\title{
LIMITS OF SUCCESSIVE CONVOLUTIONS
}

\author{
JOHN C. MARTIN
}

\begin{abstract}
On an arbitrary compact, zero-dimensional, Abelian group, if $\mu_{0}, \mu_{1}, \ldots$ is a sequence of probability measures, a condition on these measures is given which is necessary and sufficient for each of the sequences $\mu_{t}, \mu_{t} * \mu_{t+1}, \mu_{t} * \mu_{t+1} * \mu_{t+2}, \ldots$ of successive convolutions to converge to Haar measure in the weak-star topology. Some simple consequences of the theorem are noted.
\end{abstract}

Let $G$ be a compact, Abelian group and $\mu$ a Borel probability measure on $G$. If we denote by $\mu^{(n)}$ the $n$th convolution power of $\mu$, the following result is well known.

THEOREM [1, THEOREM 8]. $\mu^{(n)}$ converges to the normalized Haar measure $\lambda_{G}$ in the weak-star topology if and only if the support of $\mu$ is not contained in a coset of any proper closed subgroup of $G$.

In this paper we shall be concerned primarily with zero-dimensional, compact, Abelian groups. For such a group $G$ we consider, in place of $\mu^{(n)}$, successive convolutions of the form $\mu_{k} * \mu_{k+1} * \cdots * \mu_{k+m}$ (henceforth abbreviated $\mu_{k, m}$ ), where $\mu_{1}, \mu_{2}, \ldots$ is any sequence of Borel probability measures on $G$; we derive a necessary and sufficient condition for $\mu_{k, m}$ to converge to $\lambda_{G}$ in the weak-star topology for every $k$.

For background material, including a more complete discussion of convolutions, the reader is referred to [3].

The author wishes to thank Professor William Veech for advice regarding the preparation of this paper, and the referee for suggestions which led to a simplification of the proof.

If $G$ is any compact, Abelian group, $C(G)$ will denote the Banach space of continuous complex-valued functions on $G$ and $M(G) \subseteq C^{*}(G)$ the set of Borel probability measures on $G$. For $\mu, \nu \in M(G)$, the convolution $\mu * \nu$ is defined by

$$
\int_{G} f(x) d(\mu * \nu)(x)=\int_{G} \int_{G} f(x+y) d \mu(x) d \nu(y) \quad(f \in C(G)) .
$$

Presented to the Society, January 22, 1976 under the title Convolutions on compact zerodimensional Abelian groups; received by the editors September 30, 1975.

AMS (MOS) subject classifications (1970). Primary 43A05; Secondary 28A10.

Key words and phrases. Compact zero-dimensional Abelian group, probability measure, convolution, weak-star topology, Fourier-Stieltjes transform. 
Now if $H$ is a subgroup of $G, H$ is open and closed if and only if the index $(G: H)$ of $H$ in $G$ is finite; if $G$ is zero-dimensional, this will be true whenever $H$ is the kernel of a continuous character on $G$. Let $H$ be an open-closed subgroup, and let $\mu \in M(G)$. We define $\mu^{H} \in M(G / H)$ as follows:

$$
\mu^{H}\{[x]\}=\mu([x]) \quad([x] \in G / H) .
$$

Then it may be verified that for $\mu, \nu \in M(G)$,

$$
(\mu * \nu)^{H}=\mu^{H} * \nu^{H}
$$

We denote by $m_{H}(\mu)$ the maximum of the numbers $\mu^{H}\{[x]\}$.

Theorem. Let $G$ be a compact, zero-dimensional, Abelian group, and let $\mu_{1}, \mu_{2}, \ldots \in M(G)$. Then $\lim _{m \rightarrow \infty} \mu_{k, m}=\lambda_{G}$ in the weak-star topology for every $k$ if and only if

(2) for every proper open-closed subgroup $H$ of $G, \prod_{t=1}^{\infty} m_{H}\left(\mu_{t}\right)=0$.

REMARK. This result, stated in a different form and without reference to convolutions, is obtained in Lemma 3 of [2] in the case where $G$ is the group of integers modulo two. In this case there is a simple proof involving the formula

$$
\left|\mu_{k, m}\{0\}-\mu_{k, m}\{1\}\right|=\prod_{t=k}^{k+m}\left|\mu_{t}\{0\}-\mu_{t}\{1\}\right| .
$$

Our proof uses two preparatory lemmas.

LemMa 1. For any compact, Abelian group $G$ and any sequence $\mu_{1}, \mu_{2}, \ldots$ $\in M(G), \mu_{k, m}$ converges to $\lambda_{G}$ in the weak-star topology for every $k$ if and only if $\prod_{t=k}^{\infty}\left|\hat{\mu}_{t}(\gamma)\right|=0$ for each $k$ and each nontrivial character $\gamma$ on $G$.

Proof. It is well known that a sequence $\nu_{n}$ in $M(G)$ converges to $\nu \in M(G)$ in the weak-star topology if and only if $\hat{\nu}_{n}$ converges pointwise to $\hat{\nu}$. We have $\hat{\lambda}_{G}(1)=1, \hat{\mu}_{t}(1)=1$ for each $t$, and $\hat{\lambda}_{G}(\gamma)=0$ for $\gamma \neq 1$. The lemma follows from the fact that $\hat{\mu}_{k, m}=\hat{\mu}_{k} \hat{\mu}_{k+1} \cdots \hat{\mu}_{k+m}$. Q.E.D.

Lemma 2. Suppose $r_{i} \geqslant 0(i=0,1, \ldots, j-1)$ and $\sum_{i=0}^{j-1} r_{i}=1$. Let $R$ $=\max r_{i}$, and denote by $w$ the $j$ th root of unity $e(1 / j)=\exp (2 \pi i / j)$. Then

$$
\left|\sum_{i=0}^{j-1} r_{i} w^{i}\right| \leqslant|R+(1-R) w|
$$

The proof is straightforward.

Proof of THEOREM. Suppose (2) holds. (In fact, we need only assume that it holds for subgroups $H$ which are kernels of continuous characters on $G$.) Let $\gamma$ be any continuous character other than 1 ; let $\gamma(G)=\left\{1, w, \ldots, w^{j-1}\right\}$ (where $w=e(1 / j)$ ), and let $H=\operatorname{Ker} \gamma$. Then for $t \geqslant 1$, 


$$
\hat{\mu}_{t}(\gamma)=\int_{G} \overline{\gamma(x)} d \mu_{t}(x)=\sum_{i=0}^{j-1} r_{t, i} w^{i}
$$

where $r_{t, i}=\mu_{t}\left(\gamma^{-1}\left(w^{-i}\right)\right)$. Let $R_{t}=\max _{i} r_{t, i}=m_{H}\left(\mu_{t}\right)$. Then from Lemma 2,

$$
\left|\hat{\mu}_{t}(\gamma)\right| \leqslant\left|R_{t}+\left(1-R_{t}\right) w\right| \leqslant\left(1-2 R_{t}\left(1-R_{t}\right)(1-\cos (2 \pi / j))\right)^{1 / 2} .
$$

By Lemma 1, it is sufficient to show that

$$
\prod_{t=k}^{\infty}\left(1-2 R_{t}\left(1-R_{t}\right)(1-\cos (2 \pi / j))\right)=0 \quad(k>0),
$$

which will be true if $\sum_{t=1}^{\infty} R_{t}\left(1-R_{t}\right)$ diverges. But since $R_{t} \geqslant 1 / j$, the conclusion follows from (2).

The proof in the other direction holds for any compact Abelian group and any closed subgroup $H$ and is a straightforward application of (1). Q.E.D.

A few observations on the theorem are in order. First, it is easy to see that in the special case where $\mu_{1}=\mu_{2}=\cdots=\mu$, condition (2) reduces to the statement in the theorem of Kawada and Itô at the beginning of the paper.

We consider next the special case in which

$$
d \mu_{t}=f_{t} d \lambda_{G}
$$

where $f_{t} \in L^{2}\left(G, \lambda_{G}\right), f_{t} \geqslant 0$, and $\int_{G} f_{t} d \lambda_{G}=1$. Then since $f_{t} \in L^{2}$, each of the sequences $f_{k} * f_{k+1}, f_{k} * f_{k+1} * f_{k+2}, \ldots$ is equicontinuous and therefore has a uniformly convergent subsequence. It follows that each of these sequences converges uniformly to 1 if and only if, for every proper openclosed subgroup $H, \prod_{t=1}^{\infty} m_{H}\left(f_{t}\right)=0$ (where $\left.m_{H}\left(f_{t}\right)=m_{H}\left(\mu_{t}\right)\right)$.

Finally, we note that if (2) holds for any compact Abelian group $G$, then $\mu_{k, m}(f)$ converges to $\lambda_{G}(f)$ for each $k$, provided that $f$ is a continuous function which is constant on cosets of some open-closed subgroup; zerodimensionality assures that all trigonometric polynomials have this property.

One can consider the same question if the group $G$ is non-Abelian. In this case, the statement of the theorem will require at least some modification. Clearly, a necessary condition for $\mu_{k, m}$ to converge to $\lambda_{G}$ for each $k$ is that $\prod_{t=1}^{\infty} \mu_{t}(H)=0$ for every proper open-closed subgroup $H$; hence, the condition that (2) hold for every proper, normal, open-closed subgroup is not sufficient (though it is necessary). On the other hand, condition (2) as stated is not in general necessary: if $G$ is the permutation group $S_{3}, H=\{e, \phi\}$ is a subgroup of order $2, \psi \notin H$, and $\mu\{\psi\}=\mu\{\psi \phi\}=\frac{1}{2}$, we see that $\mu^{(n)}$ converges to $\lambda_{G}$ although the measure of the left coset $\psi H$ is 1 .

\section{REFERENCES}

1. Y. Kawada and K. Itô, On the probability distribution on a compact group. I, Proc. Phys.Math. Soc. Japan (3) 22 (1940), 977-998. MR 2, 223.

2. M. Keane, Generalized Morse sequences, Z. Wahrscheinlichkeitstheorie und Verw. Gebiete 10 (1968), 335-353. MR 39 \#406.

3. W. Rudin, Fourier analysis on groups, Interscience, New York, 1962. MR 27 \#2808.

Department of Mathematics, North Dakota State University, Fargo, North Dakota 58102 